\title{
A Respective Observational Study of Patients on Maintenance Hemodialysis Receiving Parathyroidectomy By Ultrasonic Scalpel
}

\section{Dan Gao}

Hubei University of Arts and Science

\section{Fengqi Hu}

Hubei University of Arts and Science

Zhao Gao ( $\sim$ gaozhao1648@163.com )

Hubei University of Arts and Science

\section{Hai Yuan}

Hubei University of Arts and Science

\section{Research Article}

Keywords: Haemodialysis, Chronic kidney disease, Secondary hyperparathyroidism, Parathyroidectomy, Ultrasonic scalpel

Posted Date: December 2nd, 2021

DOI: https://doi.org/10.21203/rs.3.rs-1124631/v1

License: (9) This work is licensed under a Creative Commons Attribution 4.0 International License. Read Full License 


\section{Abstract \\ Background}

Secondary hyperparathyroidism (SHPT) remains a common complication in many patients on maintenance hemodialysis. Kidney Disease Improve Global Outcomes (KDIGO) 2017 guidelines suggest that parathyroidectomy (PTX) should be performed in severe SHPT patients with chronic kidney disease stage 3a-stage 5D. In the present study, we observed the efficacy of ultrasonic scalpel for PTX in SHPT patients on maintenance hemodialysis.

\section{Methods}

A total of 74 patients on maintenance hemodialysis who underwent PTX (34 with traditional electrocautery and 40 with an ultrasonic scalpel) were observed between August 2020 and August 2021 at Xiangyang Central Hospital (Hubei University of Arts and Science). Baseline demographic and clinic characteristics were collected pre- and post-PTX. Moreover, the postoperative complications and operation time were assessed between the two groups.

\section{Results}

The univariate analysis showed that there was no statistical significance in weight, dialysis duration, serum potassium, serum calcium, alkaline phosphate, triglyceride, and intact parathyroid hormone (iPTH) before and after PTX between the two groups $(P>0.05)$. The operation time in the ultrasonic scalpel group was significantly decreased compared with the traditional electrocautery group $(P<0.05)$. Compared with the traditional electrocautery group, the drainage amount was significantly reduced in the ultrasonic scalpel group, and the number of days with drain and postoperative hospital stay were also remarkably decreased $(P<0.05)$. In addition, a higher incidence of recurrent laryngeal nerve injury occurred in the traditional electrocautery group, while the difference was not significant $(P>0.05)$.

\section{Conclusions}

The use of ultrasonic scalpel significantly reduced the operation time and postoperative hospital stay in patients on maintenance hemodialysis undergoing PTX.

\section{Introduction}

Secondary hyperparathyroidism (SHPT) has a high prevalence in patients on maintenance hemodialysis. The high level of parathyroid hormone (PTH) in SHPT patients is closely associated with mineral and bone disorders and mortality [1-3]. Although calcimimetics and vitamin D are widely used in SHPT patients on maintenance hemodialysis, some SHPT patients are refractory to medical treatments. Kidney 
Disease Improve Global Outcomes (KDIGO) 2017 guidelines suggest that parathyroidectomy (PTX) can be performed in severe SHPT patients with chronic kidney disease (CKD) stage 3a to stage 5D who fail to respond to medical therapy [4]. Therefore, PTX is required for SHPT patients who are resistant to medical therapy.

Bleeding is a common complication after thyroid or parathyroid operation $[5,6]$. The traditional operations include blood vessel ligature and electrocoagulation. However, the operation time is prolonged, and the adjacent tissues are damaged due to thermal damage of electrocoagulation. Recently, an ultrasonic scalpel that employs mechanical vibration and can simultaneously cut and coagulate tissues has been used in several surgical operations $[7,8]$.

In the present study, we aimed to assess the value of ultrasonic scalpel in PTX. In addition, postoperative complications were observed between the electrocautery and ultrasonic scalpel groups.

\section{Material And Methods}

\section{Patients}

A total of 74 SHPT patients on maintenance hemodialysis were included in this analysis. Patients with heart failure, pulmonary insufficiency, or coagulation disorders were excluded. Written informed consents were obtained from all participants.

\section{Surgery}

A total of 34 patients underwent PTX by traditional electrocautery (ERBE, VIO 300D) from August 2020 to December 2020. Since January 2021, ultrasonic scalpels became available, and 40 patients received PTX using an ultrasonic scalpel (Ultracision, Ethicon Endo-Surgery Europe).

\section{Statistical analysis}

Continuous variables were presented as mean \pm standard deviation and tested by a Mann-Whitney U-test and a Kruskal-Wallis test. Categorical variables were presented as frequency and tested by Pearson's chisquare test. The data were analyzed by SPSS 20.0. P values $<0.05$ were considered statistically significant.

\section{Results}

An ultrasonic scalpel was effectively and safely used in PTX (Fig. 1). The removed parathyroid glands should be confirmed by pathological examination (Fig. 2). The levels of PTH were usually declined to normal levels after PTX, indicating a successful operation.

Table 1 shows that the factors, such as median age, months on dialysis, body weight, and biochemical analysis, in both groups of patients were not significantly different before PTX. The levels of serum 
phosphorus, serum calcium, and intact parathyroid hormone (iPTH) in both groups were significantly decreased after PTX. The level of alkaline phosphate in both groups was significantly increased after PTX. However, there was no difference in terms of serum phosphorus, serum calcium, iPTH, and alkaline phosphate between the two groups after PTX (Table 2).

Operation time and drainage amount were significantly decreased in the ultrasonic scalpel group compared with the electrocautery group $(94.00 \pm 16.84$ vs. $123.00 \pm 25.16 ; 119.31 \pm 51.77$ vs. 145.58 46.83 , respectively). Moreover, the number of days with drain and postoperative hospital stay were significantly reduced in the ultrasonic scalpel group compared with the electrocautery group $(4.40 \pm 1.62$ vs. $5.80 \pm 2.14 ; 8.60 \pm 3.65$ vs. $10.70 \pm 4.21$, respectively). In addition, there was no difference in postoperative complications between the two groups (Table 3 ).

\section{Discussion}

Parathyroid glands are usually located on the back of the thyroid and near the recurrent laryngeal nerve. To remove parathyroid glands, the thyroid must be turned up. Postoperative bleeding is usually a common complication because the thyroid is highly vascularized. In addition, serious postoperative bleeding is life-threatening because of neck hematoma [5]. Effective intraoperative hemostasis is important in neck surgery. The common complications of PTX are bleeding, infection, and hypocalcemia $[9,10]$. To decrease the frequency of surgical complications, we observed the effects of traditional electrocautery and ultrasonic scalpels in this study.

The results of our study demonstrated that the operation time and drainage amount were significantly decreased in the ultrasonic scalpel group compared with the electrocautery group. It is well known that ultrasonic scalpel transforms electric energy into mechanical vibration. An ultrasonic scalpel can lead to vaporization, protein coaptation, and protein denaturation at a temperature lower than $100{ }^{\circ} \mathrm{C}$. However, electrocautery leads to the carbonization of tissue and reaches temperatures as high as $400{ }^{\circ} \mathrm{C}$ [11]. In addition, the number of days with drain and postoperative hospital stay were shorter in the ultrasonic scalpel group. Therefore, an ultrasonic scalpel could decrease the operation time and complications. The incidence of recurrent laryngeal nerve injury and postoperative infection in the electrocautery group was higher compared with the ultrasonic scalpel group. However, there were no significant differences between the two groups. Obviously, tissue damage was less in ultrasonic scalpel operation because of lower temperature and shorter operation time compared with the traditional operation.

Bipolar and monopolar cautery, vascular ligations, and hemostatic clips are used for hemostasis in conventional operation. These conventional techniques are often considered reliable methods for intraoperative hemostasis. However, bipolar electrocautery has been used only for very small vessels, and vascular ligation and hemostatic clips are very time-consuming techniques. Ultrasound is a more recently developed hemostatic technique with high efficacy. Ultrasonic vessel sealing devices have been shown to reduce operation time and a variety of postoperative complications, such as thermal nerve injury during thyroidectomy [12]. 
Our study had several limitations. First, our study was not a randomized controlled study. Second, the sample size of this study was small. Therefore, large-scale randomized controlled studies are required in the future.

In conclusions, an ultrasonic scalpel was effective and safe in PTX. The use of ultrasonic scalpel significantly reduced the operation time and postoperative hospital stay in patients on maintenance hemodialysis undergoing PTX.

\section{Declarations}

\section{Ethics approval}

The study was conducted following the Declaration of Helsinki. This study was approved by the ethics committee of Xiangyang Central Hospital..

\section{Funding}

This study was financed by the Natural Science Foundation of Hubei Province (2019CFB392) and Hubei Province Health and Family Planning Scientific Research Project (WJ2019Q018).

\section{Authors' contribution}

DG wrote the manuscript. FH performed the statistical analysis. ZG supervised the composition of the paper and revised it critically for important intellectual content. All authors read and approved the final manuscript.

\section{Competing interests}

The authors have declared that there is no conflict of interest.

\section{Availability of data and materials}

The datasets used and analyzed in the current study are available from the corresponding author on reasonable request.

\section{References}

1. Block GA, Kilpatrick RD, Lowe KA, Wang W, Danese MD. CKD-mineral and bone disorder and risk of death and cardiovascular hospitalization in patients on hemodialysis. Clin J Am Soc Nephrol. 2013, 8(12):2132-40.

2. Komaba $\mathrm{H}$, Nakamura M, Fukagawa M. Resurgence of parathyroidectomy: evidence and outcomes. Curr Opin Nephrol Hypertens. 2017, 26(4):243-249. 
3. Fernández-Martín JL, Martínez-Camblor P, Dionisi MP, Floege J, Ketteler M, London G, Locatelli F, Gorriz JL, Rutkowski B, Ferreira A, Bos WJ, Covic A, Rodríguez-García M, Sánchez JE, RodríguezPuyol D, Cannata-Andia JB; COSMOS group. Improvement of mineral and bone metabolism markers is associated with better survival in haemodialysis patients: the COSMOS study. Nephrol Dial Transplant. 2015, 30(9):1542-51.

4. Kidney Disease: Improving Global Outcomes (KDIGO) CKD-MBD Update Work Group. KDIGO 2017 Clinical Practice Guideline Update for the Diagnosis, Evaluation, Prevention, and Treatment of Chronic Kidney Disease-Mineral and Bone Disorder (CKD-MBD). Kidney Int Suppl (2011). 2017, 7(1):1-59.

5. Edafe O, Cochrane E, Balasubramanian SP. Reoperation for Bleeding After Thyroid and Parathyroid Surgery: Incidence, Risk Factors, Prevention, and Management. World J Surg. 2020, 44(4):11561162.

6. Talutis SD, Drake FT, Sachs T, Rao SR, McAneny D. Evacuation of postoperative hematomas after thyroid and parathyroid surgery: An analysis of the CESQIP Database. Surgery. 2019, 165(1):250256.

7. Pelizzo MR, Sorgato N, Isabella Merante Boschin I, Marzola MC, Colletti PM, Rubello D. Does the ultrasound dissector improve parathyroid gland preservation during surgery? Eur J Surg Oncol. 2014,40(7):865-8.

8. Jiang J, Shen M, Lu H. Ultrasonic scalpel with knot tying protects parathyroid function for total thyroidectomy with central neck dissection. Gland Surg. 2020, 9(2):192-199.

9. Lim CTS, Kalaiselvam T, Kitan N, Goh BL. Clinical course after parathyroidectomy in adults with endstage renal disease on maintenance dialysis. Clin Kidney J. 2018, 11(2):265-269.

10. Eidman KE, Wetmore JB. The role of parathyroidectomy in the management of secondary hyperparathyroidism. Curr Opin Nephrol Hypertens. 2017, 26(6):516-522.

11. Materazzi G, Ambrosini CE, Fregoli L, De Napoli L, Frustaci G, Matteucci V, Papini P, Bakkar S, Miccoli P. Prevention and management of bleeding in thyroid surgery. Gland Surg. 2017, 6(5):510-515.

12. Hua N, Quimby AE, Johnson-Obaseki S. Comparing Hematoma Incidence between Hemostatic Devices in Total Thyroidectomy: A Systematic Review and Meta-analysis. Otolaryngol Head Neck Surg. 2019, 161(5):770-778.

\section{Tables}

Due to technical limitations, table 1,2,3 is only available as a download in the Supplemental Files section.

\section{Figures}



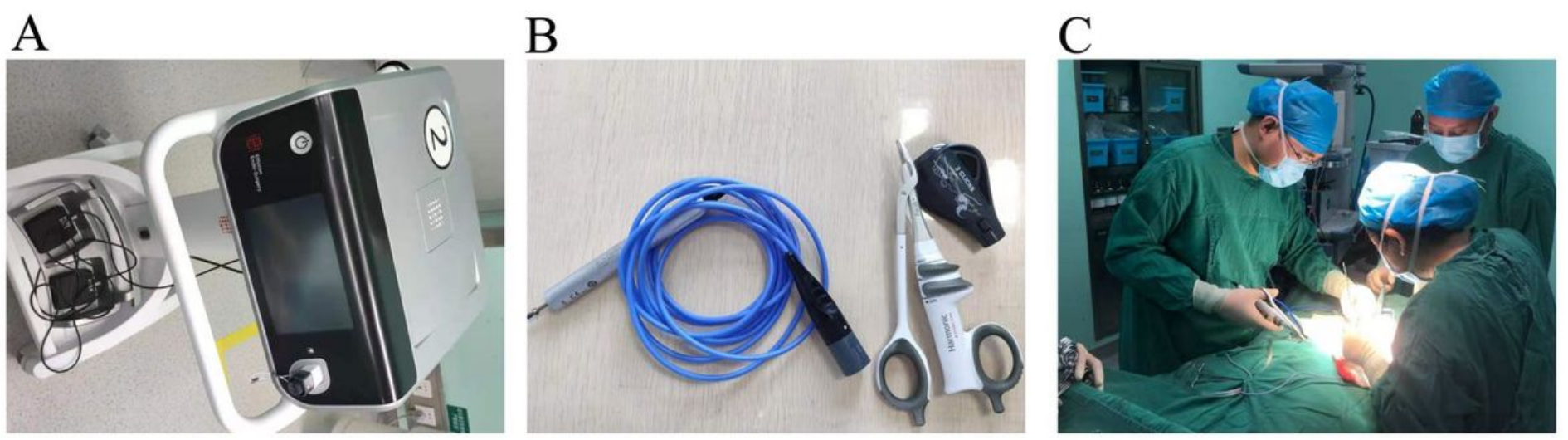

\section{Figure 1}

Ultrasonic scalpel is used in PTX (A) and (B) Ultrasonic scalpel device, (C) Ultrasonic scalpel was used in operation.
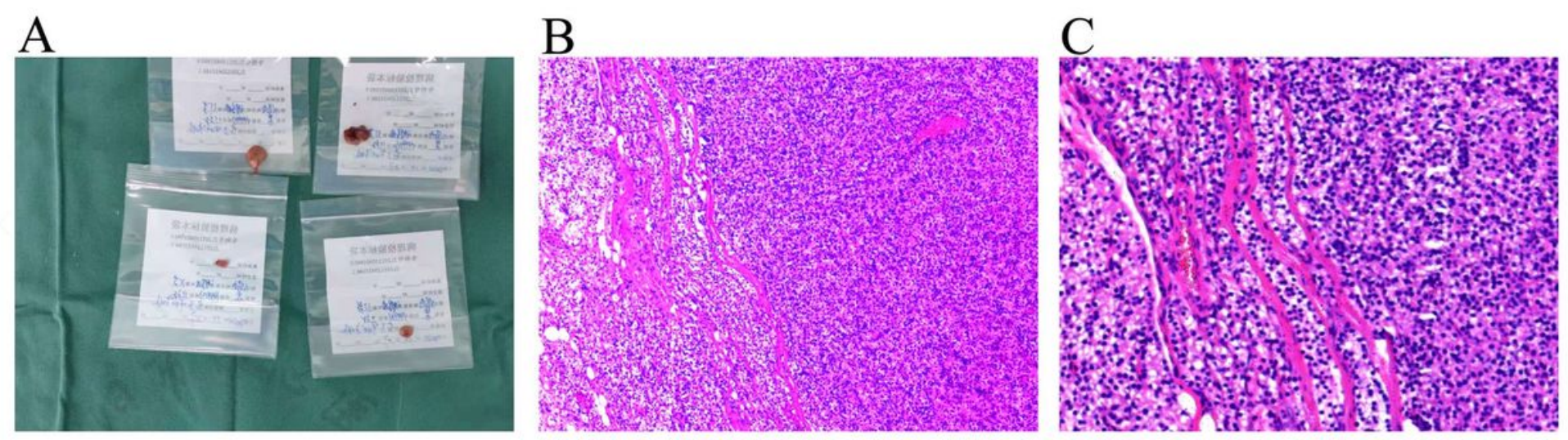

Figure 2

Parathyroid glands are analyzed by pathological examination (A) Parathyroid glands were collected during operation. (B) Representative H\&E staining of parathyroid glands. Original magnification $\times 100$. (C) Representative H\&E staining of parathyroid glands. Original magnification $\times 200$.

\section{Supplementary Files}

This is a list of supplementary files associated with this preprint. Click to download.

- Table1.jpg

- Table2.jpg 
- Table3.jpg

Page $8 / 8$ 